\title{
Temporal Patterns of Vertigo and Migraine in Vestibular Migraine
}

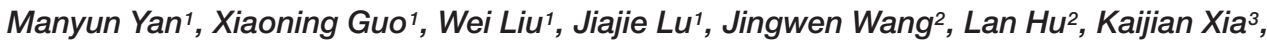 \\ Jianqiang $\mathrm{Ni}^{1}$, Haifeng $\mathrm{Lu}^{1 *}$ and Hongru $\mathrm{Zhao}^{1 *}$
}

${ }^{1}$ Department of Neurology, The First Affiliated Hospital of Soochow University, Suzhou, China, ${ }^{2}$ Department of Neurology, Ninth People's Hospital, Suzhou, China, ${ }^{3}$ Changshu No. 1 People's Hospital, Suzhou, China

Vestibular migraine (VM) is a multidisciplinary disease under exploration. Multiple temporal patterns of vertigo and migraine make it difficult to diagnose $\mathrm{VM}$, and their effect on the clinical features of VM is still unclear. Here we investigated the clinical features of VM under three temporal patterns. 172 VM patients were enrolled in this study and divided into three groups: 86 patients in group A had an earlier onset of migraine than vertigo, 35 patients in group B had an earlier onset of vertigo than migraine, and 51 patients in group $\mathrm{C}$ had concurrent vertigo and migraine. No significant difference was found among three groups regarding types, intensity and accompanying symptoms of the vestibular attack. Patients in group $\mathrm{C}$ presented higher frequency and longer duration of vertigo than group $A$ and $B$, while patients in group A presented lower frequency and shorter duration of headaches than group B and C. Additionally, the frequency, duration, intensity and accompanying symptoms of headache in group A decreased significantly after the onset of vertigo, especially in women around menopause. We hypothesized that vestibular stimulation could inhibit the trigeminal pain pathway, while painful trigeminal stimulation could excite the vestibular system. Our findings may contribute to the clinical identification of VM and further clarification of its pathogenesis.

Keywords: vestibular migraine, temporal patterns, vestibular symptoms, migraine features, interaction

\section{INTRODUCTION}

Vertigo and migraine are both very common complaints among patients, and a strong link between them has been proved. The co-occurrence of migraine and vertigo in the same individual was expected in $1 \%$ of the population based on the prevalence of migraine and vestibular vertigo, but the actual percentage was about three times higher than expected (Neuhauser et al., 2006). Moreover, migraine patients had a two- or three-fold higher risk for vertigo than those without headache (Vukovic et al., 2007). In 1999, Dieterich and Brandt introduced the term "vestibular migraine" (VM) to describe the clinical condition that associated with vestibular symptoms and migraine headache (Dieterich and Brandt, 1999). In recent years, VM, including definite VM and probable $\mathrm{VM}$, has been considered as an independent diagnostic entity and one of the most common causes of vertigo (Lempert et al., 2012; Arnold, 2018; Huang et al., 2020).

However, VM has been remained underdiagnosed despite ongoing studies in recent years. Only $8-20 \%$ of VM patients were correctly diagnosed in practice (Neuhauser et al., 2006; Geser and Straumann, 2012; Formeister et al., 2018). A big challenge for diagnosis is multiple 


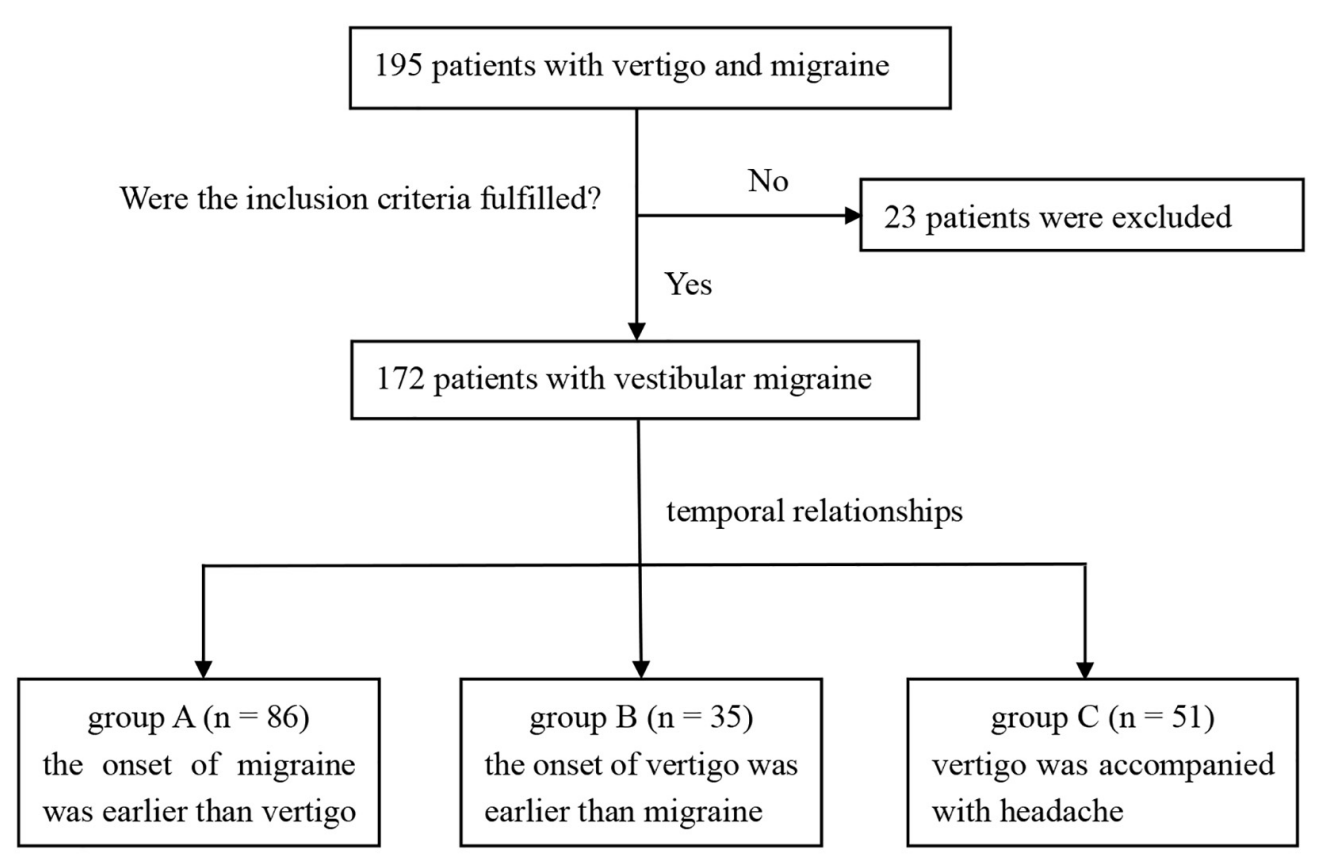

FIGURE 1 | Flow diagram of participants' selection.

temporal patterns of vertigo and migraine in VM. 51-65.6\% of VM patients suffered from migraine before the onset of vertigo (Morganti et al., 2016; Zhang et al., 2016; Beh et al., 2019), and there was an interval of $8-20$ years between the two symptoms (Cohen et al., 2011; Pagnini et al., 2014). 10.2$13 \%$ of patients had an earlier onset of vertigo than migraine (Qiu et al., 2014; Zhang et al., 2016). 34-49.6\% of patients had migraine headaches during vestibular attacks (Zhang et al., 2016; Beh et al., 2019). Since most of VM patients experienced vertigo separately from headache, and even some patients had vestibular symptoms years after the headache disappeared, they often did not actively report headache history. One study showed that only $9.8 \%$ of VM patients reported headache symptoms before being carefully asked (Ren et al., 2014). Likewise, clinicians often neglect to ask the history or associated symptoms of migraine in patients complaining with vertigo, which often results in misdiagnosis or missed diagnosis. Therefore, the investigation of temporal patterns of vertigo and migraine and their impact on patients with VM is very important for understanding and management of VM.

The aim of this study was to describe and compare the clinical features of VM patients with different temporal patterns, and investigate the interaction between vertigo and migraine headache in VM.

\section{MATERIALS AND METHODS}

\section{Participants}

Patients who met diagnostic criteria of definite VM (Arnold, 2018) or probable VM (Lempert et al., 2012) in neurology and headache clinics of First Affiliated Hospital of Soochow University from January 2018 to April 2019 were enrolled (Figure 1). The exclusion criteria were as follows: other causes of vestibular attack such as benign paroxysmal positional vertigo (BPPV), Meniere's disease or transient ischemic attack of posterior circulation; history of head trauma; severe physical illness; abnormal computed tomography or magnetic resonance imaging (MRI); history of alcohol or drugs abuse; other primary or secondary headaches; dizziness associated with chronic anxiety; history of intracranial infection.

\section{Data Collection}

A structured questionnaire was used to interview the participants regarding the following aspects: Demographics, familial history of headache or vertigo, age of onset of vertigo and migraine, vertigo intensity (Arnold, 2018) (moderate: vestibular symptoms interfere with but do not prevent daily activities; severe: daily activities cannot be continued), pain intensity [visual analog scale (VAS)], frequency (number of attacks within 3 months before enrolled), duration (minutes to hours), accompanying symptoms of vertigo (nausea, vomiting, phonophobia, photophobia, tinnitus), accompanying symptoms of headache (nausea, vomiting, phonophobia, photophobia, osmophobia, neck stiffness, scalp allodynia). Vestibular attacks included spontaneous vertigo (internal or external vertigo), positional vertigo (occurring after a change of head position), visually induced vertigo (triggered by a complex or large moving visual stimulus), head motion-induced vertigo (occurring during head motion), head motion-induced dizziness with nausea (dizziness is characterized by a sensation of disturbed spatial orientation) (Arnold, 2018). Furthermore, psychiatric comorbid 
disorders [anxiety, depression, persistent postural-perceptual dizziness (PPPD) (Staab et al., 2017) and sleep disorders] were assessed at enrollment.

We defined the temporal patterns between migraine and vertigo as follows: (A) the onset of migraine was earlier than vertigo: (i) between migraine and vertigo there was a symptomfree interval (at least one year), (ii) migraine shifted directly into vertigo without a free interval (iii) migraine gradually changed into vertigo; (B) the onset of vertigo was earlier than migraine; (C) vertigo was accompanied with headache. Moreover, headache features before the onset of vertigo in group A were additionally collected, including frequency, duration, intensity and accompanying symptoms.

\section{Statistical Analyses}

All statistical analyses were performed in SPSS software version 22.0. Categorical variables were compared using Chi-square test and presented as frequency counts and percentages. As all continuous variables in this study were non-normal distribution, they were presented as the median and interquartile range, and the Mann-Whitney $U$ test was used for comparison between two groups or the Kruskal-Wails $\mathrm{H}$ test among three groups. Statistically significance was set at a two-sided $p$-value $<0.05$.

\section{RESULTS}

172 VM patients were enrolled in this study and divided into three groups based on the temporal patterns of vertigo and migraine as shown in Figure 2. In group A, 86 patients (50.0\%) had an earlier onset of migraine than vertigo. Nine patients (5.2\%) reported vertigo attacks after the migraine headaches disappeared, with a symptom-free interval of 1-10 years. Six patients $(3.5 \%)$ reported that vertigo occurred immediately after the disappearance of headaches without any interval. 71 cases (41.3\%) reported the gradual shift of the two symptoms, with a partial overlap. In group B, 35 patients $(20.3 \%)$ had an earlier onset of vertigo than migraine. In group C, 51 patients (29.7\%) had concurrent vertigo and migraine headache. Among them, thirty patients $(17.4 \%)$ initially presented with vertigo and headache simultaneously, and 21 patients (12.2\%) complained that vertigo occurred together with headache after a period of migraine headache attacks alone.

Three groups were female-dominated, but more female subjects in group A developed vertigo around menopause than in group $\mathrm{B}(p<0.05)$ and $\mathrm{C}(p=0.084)$. Patients in group $\mathrm{B}$ presented a lower age of onset of vertigo compared to group $A$ and $\mathrm{C}(p<0.01)$ as expected. Patients in group A presented a lower age of onset of migraine compared to group B $(p<0.05)$, but similar age of onset to group C $[p$ : not significant (NS)]. The proportion of familial history of migraine in group B was lower than that in group A and C $(p<0.05)$. The proportion of motion sickness history in group $\mathrm{C}$ was higher than that in group B $(p<0.05)$ (Table 1$)$.

No significant difference was found among groups regarding types, intensity and accompanying symptoms of vestibular attack
( $p$ : NS). Patients in group C presented higher frequency and longer duration of vertigo than group A and B. (Table 2).

Patients in group A presented lower frequency and shorter duration of migraine headaches than group $\mathrm{B}$ and $\mathrm{C}(p<0.05)$ (Table 3). Furthermore, the frequency, duration, intensity and accompanying symptoms of headache in group A decreased significantly after the onset of vertigo (Table 4). Patients in group $\mathrm{C}$ presented stronger intensity of headache and reported nausea and vomiting during headache attacks more often compared to patients in group A $(p<0.001)$ and B $(p<0.05)$ (Table 3).

Following the onset of vestibular or headache symptoms, many were diagnosed with anxiety (36.6\%), depression (47.7\%), PPPD (34.9\%) and sleep disorders (70.3\%). Except that the proportion of anxiety in group $\mathrm{C}$ was higher than that in group A $(p<0.05)$, no significant difference in the proportion of psychiatric comorbid disorders was found among groups ( $p$ : NS) (Table 5).

\section{DISCUSSION}

Temporal patterns of vertigo and migraine in VM patients and how they affect the clinical features of VM have not yet been systematically described. In this study, we investigated the clinical features of VM under three temporal patterns and found that patients with vertigo and migraine occurred simultaneously presented higher frequency and longer duration of vertigo, while patients with earlier onset of migraine than vertigo presented lower frequency and shorter duration of headache.

Previous studies have suggested that headaches significantly decreased in frequency and strength or disappeared after the onset of vertigo in VM patients (von Brevern and Lempert, 2016; Teggi et al., 2018). This phenomenon was more common in women around menopause (Lempert et al., 2009; Park and Viirre, 2010). A 13-year observational study showed that headaches

TABLE 1 | Demographic data among subjects in group A, B, and C.

\begin{tabular}{|c|c|c|c|}
\hline & A $(n=86)$ & B $(n=35)$ & $C(n=51)$ \\
\hline $\begin{array}{l}\text { Age at inclusion } \\
\text { (years) }\end{array}$ & $49(37,59)$ & $43(33,60)$ & $47(33,57)$ \\
\hline Sex (female \%) & $69(80 \%)$ & 30 (86\%) & $41(80 \%)$ \\
\hline $\begin{array}{l}\text { Vertigo occurred } \\
\text { around menopause } \\
\text { (of females) }\end{array}$ & $28(41 \%)$ & $5(17 \%)^{\star}$ & $10(24 \%)$ \\
\hline $\begin{array}{l}\text { Age of onset of } \\
\text { vertigo (years) }\end{array}$ & $43(33,54)$ & $29(22,37)^{\star}$ & $40(30,49)+$ \\
\hline $\begin{array}{l}\text { Age of onset of } \\
\text { migraine (years) }\end{array}$ & $32(25,41)$ & $37(31,42)^{\star}$ & $32(25,43)$ \\
\hline $\begin{array}{l}\text { Familial history of } \\
\text { vertigo }\end{array}$ & $21(24 \%)$ & $9(26 \%)$ & 18 (35\%) \\
\hline $\begin{array}{l}\text { Familial history of } \\
\text { migraine }\end{array}$ & $36(42 \%)$ & $7(20 \%)^{\star}$ & $21(41 \%)+$ \\
\hline $\begin{array}{l}\text { Motion sickness } \\
\text { history }\end{array}$ & 50 (58\%) & 17 (49\%) & 37 (73\%)† \\
\hline
\end{tabular}

A: the onset of migraine was earlier than vertigo; $B$ : the onset of vertigo was earlier than migraine; C: vertigo was accompanied with headache. ${ }^{*} p<0.05$ vs. group $A$; $+p<0.05$ vs. group $B$. 

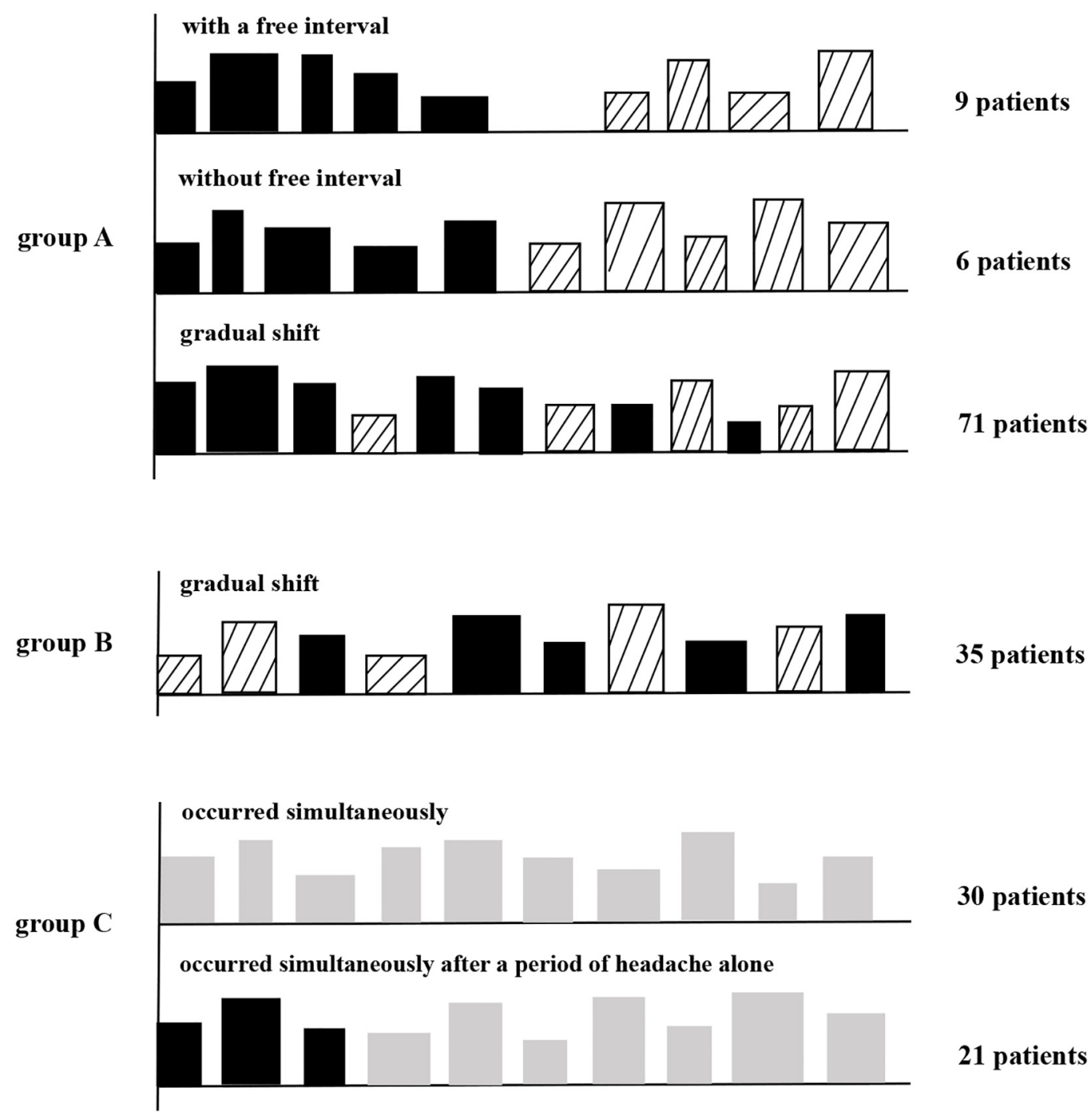

\section{0 patients}

\section{1 patients}

FIGURE 2 | Schematic diagram of temporal patterns of vertigo and migraine in VM patients. Group A, patients had an earlier onset of migraine than vertigo; group B, patients had an earlier onset of vertigo than migraine; and group C, patients had concurrent vertigo and migraine. Height and width of rectangles indicate, respectively, the strength and duration of headache or vertigo.

completely ceased after vertigo attacked in 57\% of patients and substantially improved in 43\% (Pagnini et al., 2014). However, there were no detailed data to clarify the specific changes in migraine headache features before and after the onset of vertigo. In our cases, we noticed a 50\% reduction in frequency and duration of headache after the onset of vertigo, a $17 \%$ reduction in pain intensity and a $22-50 \%$ reduction in accompanying symptoms such as nausea, vomiting, photophobia, phonophobia, osmophobia, neck stiffness, and scalp allodynia. The difference between our study and the previous study (Pagnini et al., 2014) was that only 19 patients (22\%) reported complete cessation of headache in this study, $35 \%$ noticed a considerable reduction in frequency of headache after vertigo, 23\% got relief for duration, intensity or accompanying symptoms, but in $20 \%$ cases, headache symptoms were unaffected by vertigo, which was partly due to the different inclusion criteria. We included all patients diagnosed with VM, and the previous study (Pagnini et al., 2014) only included patients who developed vestibular symptoms after the disappearance or remarkable reduction of headache. Another reason was that this study was not a longitudinal study, and there was recall bias in the features of migraine headache prior to the onset of vertigo.

The mechanisms of disappearance or attenuation of headache after recurrent vertigo in VM are unclear. We hypothesized 
TABLE 2 | Vestibular symptoms among subjects in group A, B, and C.

\begin{tabular}{|c|c|c|c|}
\hline Vestibular symptoms & A $(n=86)$ & B $(n=35)$ & $C(n=51)$ \\
\hline \multicolumn{4}{|l|}{ Vestibular attack } \\
\hline Spontaneous vertigo & $38(44 \%)$ & $14(40 \%)$ & $15(29 \%)$ \\
\hline Positional vertigo & $26(30 \%)$ & $11(31 \%)$ & 15 (29\%) \\
\hline $\begin{array}{l}\text { Head motion dizziness with } \\
\text { nausea }\end{array}$ & $24(28 \%)$ & 12 (34\%) & $22(43 \%)$ \\
\hline $\begin{array}{l}\text { Head-motion vertigo or } \\
\text { visual vertigo }\end{array}$ & $4(5 \%)$ & $1(3 \%)$ & $1(2 \%)$ \\
\hline Frequency (times/3 months) & $3(1,9)$ & $3(0.6,10.5)$ & $6(3,12)^{\star}+$ \\
\hline Duration (h) & $2(0.3,18.5)$ & $2.5(0.5,30)$ & $18(2.5,24)^{\star}$ \\
\hline $5 \mathrm{~min}-1 \mathrm{~h}$ & 33 (38\%) & $14(40 \%)$ & $9(18 \%)^{*}+$ \\
\hline $1-24 \mathrm{~h}$ & 38 (44\%) & $13(37 \%)$ & 25 (49\%) \\
\hline $24-72 \mathrm{~h}$ & $15(17 \%)$ & $8(23 \%)$ & $17(33 \%)^{*}$ \\
\hline Intensity (severe \%) & 33 (38\%) & $11(31 \%)$ & $13(26 \%)$ \\
\hline \multicolumn{4}{|l|}{$\begin{array}{l}\text { Accompanying } \\
\text { symptoms }\end{array}$} \\
\hline Nausea & $61(71 \%)$ & $20(57 \%)$ & 37 (73\%) \\
\hline Vomiting & $51(59 \%)$ & $17(49 \%)$ & $26(51 \%)$ \\
\hline Photophobia & $42(49 \%)$ & $18(51 \%)$ & $21(41 \%)$ \\
\hline Phonophobia & $57(66 \%)$ & $23(66 \%)$ & $33(65 \%)$ \\
\hline Tinnitus & $20(23 \%)$ & $6(17 \%)$ & $11(22 \%)$ \\
\hline
\end{tabular}

A: the onset of migraine was earlier than vertigo; $B$ : the onset of vertigo was earlier than migraine; $C$ : vertigo was accompanied with headache. ${ }^{*} p<0.05$ vs. group $A$; tp $<0.05$ vs. group $B$.

TABLE 3 | Headache symptoms among subjects in group A, B, and C.

\begin{tabular}{|c|c|c|c|}
\hline Headache symptoms & A $(n=86)$ & B $(n=35)$ & $C(n=51)$ \\
\hline Frequency (times/3 months) & $3(0.3,7.9)$ & $6(3,12)^{*}$ & $7.5(3,16.5)^{\star}$ \\
\hline Duration (h) & $12(2,24)$ & $24(5,36)^{\star}$ & $24(4.5,36)^{\star}$ \\
\hline Intensity (VAS) & $5(3,6)$ & $5(4,7)$ & $5(5,7)^{\star} \dagger$ \\
\hline \multicolumn{4}{|l|}{ Accompanying symptoms } \\
\hline Nausea & $34(40 \%)$ & $18(51 \%)$ & $38(75 \%)^{\star} \dagger$ \\
\hline Vomiting & $20(23 \%)$ & $10(29 \%)$ & $27(53 \%)^{\star}+$ \\
\hline Photophobia & $26(30 \%)$ & $17(49 \%)$ & $21(41 \%)$ \\
\hline Phonophobia & 47 (55\%) & $23(66 \%)$ & $35(69 \%)$ \\
\hline Osmophobia & $4(5 \%)$ & $4(11 \%)$ & $6(12 \%)$ \\
\hline Neck stiffness & $13(15 \%)$ & $9(26 \%)$ & $14(27 \%)$ \\
\hline Scalp allodynia & $14(16 \%)$ & 5 (14\%) & $5(10 \%)$ \\
\hline
\end{tabular}

A: the onset of migraine was earlier than vertigo; $B$ : the onset of vertigo was earlier than migraine; C: vertigo was accompanied with headache. VAS: visual analog scale. ${ }^{*} p<0.05$ vs. group $A ;+p<0.05$ vs. group $B$.

that one potential mechanism was the interaction of the vestibular and trigeminal systems. The main mechanism of $\mathrm{VM}$ is currently considered as the connection of trigeminal caudal nucleus with vestibular nucleus (Furman et al., 2013; Huang et al., 2020), so the vestibular stimulation and migraine headache could interact with each other. A study showed that headaches completely disappeared or reduced in strength after vestibular thermal stimulation during migraine attacks (Kolev, 1990), which suggested that the painful conduction pathway might be inhibited after the activation of the vestibular pathway. On the other hand, both the prevalence of migraine and VM was significantly higher in females than males, and
TABLE 4 | Headache symptoms before and after the onset of vertigo in group A.

\begin{tabular}{lccc}
\hline Headache symptoms & Before $(\boldsymbol{n}=\mathbf{8 6})$ & After $(\boldsymbol{n}=\mathbf{8 6})$ & $\boldsymbol{p}$-value \\
\hline Frequency (times/3 months) & $6(3,9)$ & $3(0,8)$ & 0.015 \\
Duration (h) & $24(7.5,36)$ & $12(2,24)$ & 0.001 \\
Intensity (VAS score) & $6(5,7)$ & $5(3,6)$ & $<0.001$ \\
Accompanying & & & \\
symptoms & & $34(40 \%)$ & 0.126 \\
Nausea & $24(51 \%)$ & $20(23 \%)$ & 0.128 \\
Vomiting & $38(44 \%)$ & $26(30 \%)$ & 0.058 \\
Photophobia & $60(70 \%)$ & $47(55 \%)$ & 0.041 \\
Phonophobia & $8(9 \%)$ & $4(5 \%)$ & 0.369 \\
Osmophobia & $23(27 \%)$ & $13(15 \%)$ & 0.061 \\
Neck stiffness & $22(26 \%)$ & $14(16 \%)$ & 0.134 \\
Scalp allodynia & $2(2,4)$ & $2(0,3)$ & 0.002 \\
Number of accompanying & & & \\
symptoms & & & \\
\hline
\end{tabular}

Group A: the onset of migraine was earlier than vertigo; VAS: visual analog scale.

TABLE 5 | Psychiatric comorbid disorders among subjects in group A, B, and C.

\begin{tabular}{llll}
\hline Disorders & A $(\boldsymbol{n}=\mathbf{8 6})$ & B $(\boldsymbol{n}=\mathbf{3 5})$ & $\mathbf{C}(\boldsymbol{n}=\mathbf{5 1})$ \\
\hline Anxiety & $24(28 \%)$ & $13(37 \%)$ & $26(51 \%)^{\star}$ \\
Depression & $40(47 \%)$ & $15(43 \%)$ & $27(53 \%)$ \\
PPPD & $30(35 \%)$ & $15(43 \%)$ & $15(29 \%)$ \\
Sleep disorders & $56(65 \%)$ & $29(83 \%)$ & $36(71 \%)$ \\
\hline
\end{tabular}

A: the onset of migraine was earlier than vertigo; $B$ : the onset of vertigo was earlier than migraine; C: vertigo was accompanied with headache. PPPD: persistent postural-perceptual dizziness. ${ }^{*} p<0.05$ vs. group A.

the conversion from headache to vertigo was more common around menopause (Lempert et al., 2009; Park and Viirre, 2010; Pagnini et al., 2014), which has been confirmed in our data. Moreover, migraine tended to improve when sex hormone levels stabilized after menopause (Vetvik and MacGregor, 2017), and hormone fluctuations during the perimenopausal period were associated with an increased risk of vertigo (Park and Viirre, 2010). Hence, another possible hypothesis was the role of female sex hormones as a facilitating factor for the transformation of migraine headaches into vestibular attacks.

An interesting aspect verified in our study was that VM patients with vertigo and headache occurred simultaneously presented higher frequency and longer duration of vertigo. Similarly, this phenomenon might be due to the interaction of the vestibular and trigeminal systems. A study showed that trigeminal stimulation induced nystagmus in patients with migraine but not controls, which suggested increased vestibular excitability in migraine patients (Marano et al., 2005). Functional imaging of the brain showed that the activation of temporoparieto-insular areas and bilateral thalami was increased during vertigo attacks in VM patients (Shin et al., 2014), and the magnitude of thalamic activation was positively correlated with the frequency of migraine attacks (Russo et al., 2014). Based on this, we hypothesized that vestibular excitability increased when vertigo occurred together with migraine headaches, and the thalamus and other brain regions might be activated more 
significantly after both vestibular and painful stimulation. That is to say, painful trigeminal stimulation might enhance the excitability of the vestibular system, and the threshold of vestibular perception of VM patients decreases and the sensitivity increases, similar to the central sensitization effect. In addition, patients with concurrent vertigo and headache experienced severer headaches accompanied with nausea and vomiting more often, which might be associated with more frequent attacks of vertigo. But no significant correlations were found between other clinical features of migraine (e.g., disease duration, pain intensity, migraine disability) and thalamic activation, except for the frequency (Russo et al., 2014). And the influence of migraine symptoms on vestibular attacks remains to be further studied. Other factors that should be considered were the contributions of motion sickness history and anxiety, which were more common in VM patients with concurrent vertigo and migraine. Anxiety was associated with significantly increased recurrence of VM (Formeister et al., 2018) and motion sickness could enhance motion intolerance (Wang and Lewis, 2016).

The special temporal patterns of vertigo and migraine make it difficult for many clinicians to accurately diagnose VM, and patients usually undergo a painful and protracted course until they visit a headache or vertigo specialist. During the course, VM patients are usually anxious about unexpected and intense vertigo attacks, afraid of falling and avoid going out or entering various environments triggering dizziness (Kutay et al., 2017). Additionally, many of them complain of decreased daily activities and nearly constant dizziness and lightheaded (von Brevern and Lempert, 2016). Many studies suggested that VM patients were prone to psychiatric comorbidities, such as anxiety (19.8-70.2\%) (Langhagen et al., 2014; Beh et al., 2019), depression (21.8-40.5\%) (Vuralli et al., 2018; Beh et al., 2019), PPPD (32.8-41\%) (Neff et al., 2012; Eggers et al., 2014; Beh et al., 2019) and sleep disorders (29-67.4\%) (Vuralli et al., 2018; Beh et al., 2019; Wu et al., 2019), regardless of the frequency of vertigo. Our study showed that VM patients also had high rates of psychiatric comorbidities regardless of the temporal patterns. Vestibular attacks increase the incidence of psychiatric comorbidities, and psychiatric disorders aggravate the severity of vestibular symptoms. Then a vicious circle is formed, and the disease becomes deferred. Therefore, we should make an effort to understand these temporal patterns in VM, in order to give patients correct diagnosis and timely treatment.

This study had several potential limitations. Firstly, we lacked data on the features of vertigo before the onset of migraine in group B to further support our hypothesis that migraine headaches could increase the vestibular excitability and aggravate the manifestations of vertigo. Moreover, we only described the clinical features of VM by a cross-sectional study. A longterm longitudinal study will be of great help to understand the temporal patterns of VM and identify this entity. Secondly, we enrolled patients with probable VM, the diagnostic criteria of which have not been included in the International Classification of Headache Disorders 3rd edition (ICHD-3). But some studies have shown that there was no significant difference between definite and probable VM (Eggers et al., 2011; Van Ombergen et al., 2015; Cho et al., 2016), and most probable VM patients would develop definite VM over time during long-term follow up (Radtke et al., 2011).

\section{CONCLUSION}

We found that the temporal patterns of vertigo and migraine affected the clinical features of VM. Migraine headaches usually disappeared or relieved after recurrent vertigo attacks, especially in women around menopause, while vestibular vertigo was more frequent and lasted longer when accompanied with migraine headaches. The underlying mechanism might be that vestibular stimulation inhibits the trigeminal pain pathway, while painful trigeminal stimulation could excite the vestibular system.

\section{DATA AVAILABILITY STATEMENT}

All datasets generated for this study are included in the article/supplementary material.

\section{ETHICS STATEMENT}

The studies involving human participants were reviewed and approved by the Institutional Review Board of The First Affiliated Hospital of Soochow University. The patients/participants provided their written informed consent to participate in this study.

\section{AUTHOR CONTRIBUTIONS}

HZ, HL, JN, and MY designed the study. HZ, MY, XG, WL, JL, $\mathrm{LH}, \mathrm{JW}$, and KX evaluated the subjects and collected the data. MY and XG analyzed the data. MY wrote the initial draft, with $\mathrm{HL}$ and $\mathrm{HZ}$ participating in revising the manuscript.

\section{FUNDING}

This study was funded by the National Natural Science Foundation of China (NSFC, No. 81701309 to HL).

\section{ACKNOWLEDGMENTS}

We would like to thank Prof. Xingshun $\mathrm{Xu}$ for valuable comments on this manuscript. 


\section{REFERENCES}

Arnold, M. (2018).Headache classification committee of the international headache society (ihs) the international classification of headache disorders, 3rd edition. Cephalalgia 38, 1-211. doi: 10.1177/0333102417738202

Beh, S. C., Masrour, S., Smith, S. V., and Friedman, D. I. (2019). The spectrum of vestibular migraine: clinical features, triggers, and examination findings. Headache 59, 727-740. doi: 10.1111/head.13484

Cho, S. J., Kim, B. K., Kim, B. S., Kim, J. M., Kim, S. K., Moon, H. S., et al. (2016). Vestibular migraine in multicenter neurology clinics according to the appendix criteria in the third beta edition of the International classification of headache disorders. Cephalalgia 36, 454-462. doi: 10.1177/0333102415597890

Cohen, J. M., Bigal, M. E., and Newman, L. C. (2011). Migraine and vestibular symptoms-identifying clinical features that predict "vestibular migraine". Headache 51, 1393-1397. doi: 10.1111/j.1526-4610.2011.01934.x

Dieterich, M., and Brandt, T. (1999). Episodic vertigo related to migraine (90 cases): vestibular migraine? J. 246, 883-892. doi: 10.1007/s004150050478

Eggers, S. D., Neff, B. A., Shepard, N. T., and Staab, J. P. (2014). Comorbidities in vestibular migraine. J. Vestib. Res. 24, 387-395. doi: 10.3233/VES- 140525

Eggers, S. D., Staab, J. P., Neff, B. A., Goulson, A. M., Carlson, M. L., and Shepard, N. T. (2011). Investigation of the coherence of definite and probable vestibular migraine as distinct clinical entities. Otol. Neurotol. 32, 1144-1151. doi: 10. 1097/MAO.0b013e31822alc67

Formeister, E. J., Rizk, H. G., Kohn, M. A., and Sharon, J. D. (2018). The epidemiology of vestibular migraine: a population-based survey study. Otol. Neurotol. 39, 1037-1044. doi: 10.1097/MAO.0000000000001900

Furman, J. M., Marcus, D. A., and Balaban, C. D. (2013). Vestibular migraine: clinical aspects and pathophysiology. Lancet Neurol. 12, 706-715. doi: 10.1016/ s1474-4422(13)70107-8

Geser, R., and Straumann, D. (2012). Referral and final diagnoses of patients assessed in an academic vertigo center. Front. Neurol. 3:169. doi: 10.3389/fneur. 2012.00169

Huang, T. C., Wang, S. J., and Kheradmand, A. (2020). Vestibular migraine: an update on current understanding and future directions. Cephalalgia 40, 107-121. doi: 10.1177/0333102419869317

Kolev, O. (1990). How caloric vestibular irritation influences migraine attacks. Cephalalgia 10, 167-169. doi: 10.1046/j.1468-2982.1990.1004167.x

Kutay, O., Akdal, G., Keskinoglu, P., Balci, B. D., and Alkin, T. (2017). Vestibular migraine patients are more anxious than migraine patients without vestibular symptoms. J. Neurol. 264(Suppl. 1), 37-41. doi: 10.1007/s00415-017-8439-6

Langhagen, T., Lehrer, N., Borggraefe, I., Heinen, F., and Jahn, K. (2014). Vestibular migraine in children and adolescents: clinical findings and laboratory tests. Front. Neurol. 5:292. doi: 10.3389/fneur.2014.00292

Lempert, T., Neuhauser, H., and Daroff, R. B. (2009). Vertigo as a symptom of migraine. Ann. N. Y. Acad. Sci. 1164, 242-251. doi: 10.1111/j.1749-6632.2009. 03852.x

Lempert, T., Olesen, J., Furman, J., Waterston, J., Seemungal, B., Carey, J., et al. (2012). Vestibular migraine: diagnostic criteria. J. Vestib. Res. 22, 167-172. doi: 10.3233/VES-2012-0453

Marano, E., Marcelli, V., Di Stasio, E., Bonuso, S., Vacca, G., Manganelli, F., et al. (2005). Trigeminal stimulation elicits a peripheral vestibular imbalance in migraine patients. Headache 45, 325-331. doi: 10.1111/j.1526-4610.2005. 05069.x

Morganti, L. O., Salmito, M. C., Duarte, J. A., Bezerra, K. C., Simoes, J. C., and Gananca, F. F. (2016). Vestibular migraine: clinical and epidemiological aspects. Braz. J. Otorhinolaryngol. 82, 397-402. doi: 10.1016/j.bjorl.2015.06.003

Neff, B. A., Staab, J. P., Eggers, S. D., Carlson, M. L., Schmitt, W. R., Van Abel, K. M., et al. (2012). Auditory and vestibular symptoms and chronic subjective dizziness in patients with Meniere's disease, vestibular migraine, and Meniere's disease with concomitant vestibular migraine. Otol. Neurotol. 33, 1235-1244. doi: 10.1097/MAO.0b013e31825d644a

Neuhauser, H. K., Radtke, A., von Brevern, M., Feldmann, M., Lezius, F., Ziese, T., et al. (2006). Migrainous vertigo: prevalence and impact on quality of life. Neurology 67, 1028-1033. doi: 10.1212/01.wnl.0000237539.09942.06

Pagnini, P., Vannucchi, P., Giannoni, B., and Pecci, R. (2014). Epigone migraine vertigo (EMV): a late migraine equivalent. Acta Otorhinolaryngol. Ital. 34, $62-70$.
Park, J. H., and Viirre, E. (2010). Vestibular migraine may be an important cause of dizziness/vertigo in perimenopausal period. Med. Hypotheses 75, 409-414. doi: 10.1016/j.mehy.2009.04.054

Qiu, F., Huang, X., Wang, X., Liu, J., and Qi, X. (2014). An analysis of clinical features of 226 vestibular migraine patients. Zhonghua Nei Ke Za Zhi 53, 961-963. doi: 10.3760/cma.j.issn.0578-1426.2014.12.009

Radtke, A., Neuhauser, H., von Brevern, M., Hottenrott, T., and Lempert, T. (2011). Vestibular migraine-validity of clinical diagnostic criteria. Cephalalgia 31, 906-913. doi: 10.1177/0333102411405228

Ren, T., Dai, C., and Wang, W. (2014). Clinical characteristics of vestibular migraine in 102 patients. Chin. J. Ophthalmol. Otorhinolaryngol. 14, 146-150. doi: 10.14166/j.issn.1671-2420.2014.03.003

Russo, A., Marcelli, V., Esposito, F., Corvino, V., Marcuccio, L., Giannone, A., et al. (2014). Abnormal thalamic function in patients with vestibular migraine. Neurology 82, 2120-2126. doi: 10.1212/WNL.0000000000000496

Shin, J. H., Kim, Y. K., Kim, H. J., and Kim, J. S. (2014). Altered brain metabolism in vestibular migraine: comparison of interictal and ictal findings. Cephalalgia 34, 58-67. doi: 10.1177/0333102413498940

Staab, J. P., Eckhardt-Henn, A., Horii, A., Jacob, R., Strupp, M., Brandt, T., et al. (2017). Diagnostic criteria for persistent postural-perceptual dizziness (PPPD): consensus document of the committee for the classification of vestibular disorders of the barany society. J. Vestib. Res. 27, 191-208. doi: 10.3233/VES170622

Teggi, R., Colombo, B., Albera, R., Asprella Libonati, G., Balzanelli, C., Batuecas Caletrio, A., et al. (2018). Clinical features of headache in patients with diagnosis of definite vestibular migraine: the VM-Phenotypes projects. Front. Neurol. 9:395. doi: 10.3389/fneur.2018.00395

Van Ombergen, A., Van Rompaey, V., Van de Heyning, P., and Wuyts, F. (2015). Vestibular migraine in an otolaryngology clinic: prevalence, associated symptoms, and prophylactic medication effectiveness. Otol. Neurotol. 36, 133138. doi: 10.1097/MAO.0000000000000596

Vetvik, K. G., and MacGregor, E. A. (2017). Sex differences in the epidemiology, clinical features, and pathophysiology of migraine. Lancet Neurol. 16, 76-87. doi: 10.1016/S1474-4422(16)30293-9

von Brevern, M., and Lempert, T. (2016). Vestibular migraine. Handb. Clin. Neurol. 137, 301-316. doi: 10.1016/B978-0-444-63437-5.00022-4

Vukovic, V., Plavec, D., Galinovic, I., Lovrencic-Huzjan, A., Budisic, M., and Demarin, V. (2007). Prevalence of vertigo, dizziness, and migrainous vertigo in patients with migraine. Headache 47, 1427-1435. doi: 10.1111/j.1526-4610. 2007.00939.x

Vuralli, D., Yildirim, F., Akcali, D. T., Ilhan, M. N., Goksu, N., and Bolay, H. (2018). Visual and postural motion-evoked dizziness symptoms are predominant in vestibular migraine patients. Pain. Med. 19, 178-183. doi: 10.1093/pm/ pnx182

Wang, J., and Lewis, R. F. (2016). Contribution of intravestibular sensory conflict to motion sickness and dizziness in migraine disorders. J. Neurophysiol. 116, 1586-1591. doi: 10.1152/jn.00345.2016

Wu, J., Liu, C., Yu, H., Li, H., Jia, Y., Zhang, D., et al. (2019). Clinical characteristics of sleep disorders in patients with vestibular migraine. Sleep Breath doi: 10.1007/ s11325-019-01994-1

Zhang, Y., Kong, Q., Chen, J., Li, L., Wang, D., and Zhou, J. (2016). International classification of headache disorders 3rd edition beta-based field testing of vestibular migraine in China: demographic, clinical characteristics, audiometric findings and diagnosis statues. Cephalalgia 36, 240-248. doi: 10. $1177 / 0333102415587704$

Conflict of Interest: The authors declare that the research was conducted in the absence of any commercial or financial relationships that could be construed as a potential conflict of interest.

Copyright (C) 2020 Yan, Guo, Liu, Lu, Wang, Hu, Xia, Ni, Lu and Zhao. This is an open-access article distributed under the terms of the Creative Commons Attribution License (CC BY). The use, distribution or reproduction in other forums is permitted, provided the original author(s) and the copyright owner(s) are credited and that the original publication in this journal is cited, in accordance with accepted academic practice. No use, distribution or reproduction is permitted which does not comply with these terms. 